\title{
Assessing diagnostic applicability of heat release characteristics determined based on ship engine indicator diagrams
}

\author{
Stanisław Polanowski, Prof. \\ Polish Naval University
}

\begin{abstract}

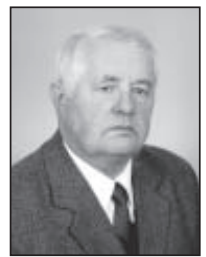

In order to determine indicator diagrams-based heat release characteristics, a single-zone model of net heat release was used for perfect gas. It was proved that when a constant value for isentropic exponent is assumed, the error in determining the characteristics can be limited to $1 \%$ at the nominal load. The effect of errors in determining the position of piston TDC, as well as that of gas passages and indicator valves on the calculated characteristics was evaluated. It was shown that for low-speed engines the effect of gas passages in negligible, while for medium-speed engines the characteristics reveal some deformations (waves), which are repeatable for an individual cylinder in the examined engine construction. The results of the performed investigations and analyses suggest possibility and advisability of the use of heat release characteristics in diagnosing ship engines, in particular low-speed machines.
\end{abstract}

Keywords: ship piston engines; indicator diagrams; heat release characteristics; diagnostic application

\section{INTRODUCTION}

Since the production of the combustion pressure analyser NK- 5 by Autronica, and the analyser Cyldet 1800 by ABB in the seventies of the last century, the range of use of the information contained in the indicator diagrams has not changed.

For the time being, the main functions executed by pressure analysers include: calculating average indicated pressure, determining maximum combustion pressure, recording TDC pressure, and the presentation of the determined values in the tabularised form and histograms, as well as the presentation and comparison of indicator diagrams on a monitor screen. Some analysers have also included an option of determining the rate of combustion pressure building up.

Diagnostic inference based on tables of parameters, histograms and comparison of indicator diagrams is rather unreliable and highly inaccurate. Hence, some monitoring and diagnostic systems have included an additional option of pressure measurement and analysis in the fuel injection system.

Perception of the diagnostic information contained in the indicator diagram can be extended by making use of heat release characteristics determined based on the indicator diagrams.

\section{SELECTING THE HEAT RELEASE MODEL}

It is Paul Henry Schweitzer [5] who is believed to be the author of the first heat release model for the Diesel engine [1, 4]. Remarkable development of advanced calculation models, observed since the late fifties of the last century, is connected with the appearance of possibilities for computer aided data processing and computer simulations.

At present, model investigations of combustion processes make use of complicated, multidimensional models of heat release processes. For instance, most advanced models include simulation programmes KIVA worked out in the Los Alamos National Laboratory. These model are characterised by a large number of immeasurable quantities and parameters, and their use requires high computational potential.

The simplest and most frequently used model which calculates heat release in a Diesel engine based on indicator diagrams is believed to be the single-zone model developed by Krieger and Borman $[1,4]$. It is assumed in this model that at each time instant the working medium (charge), having the form of uniform mixture of air and combustions products, is in the state of thermodynamic equilibrium.

The first law of thermodynamics in the differential form for an open system can be written as:

$$
\mathrm{dQ}_{\mathrm{sp}}-\mathrm{dQ}_{\mathrm{ch}}-\mathrm{dQ}_{\mathrm{r}}=\mathrm{dQ}_{\mathrm{n}}=\mathrm{dU}+\mathrm{dW}
$$

where:

$\mathrm{dQ}_{\mathrm{sp}} \quad$ - heat released as a result of fuel combustion

$\mathrm{dQ}_{\mathrm{ch}} \quad$ - heat of cooling

$\mathrm{dQ}_{\mathrm{r}} \quad$ - heat lost as a result of transfer of substances through system boundaries

$\mathrm{dQ}_{\mathrm{n}} \quad-$ net heat release

$\mathrm{dU}^{\mathrm{n}} \quad$ - internal energy of the charge

$\mathrm{dW} \quad-$ work done by the system. 
The heat $\mathrm{Q}_{\mathrm{r}}$ is the result of fuel flow to the cylinder and gas scavenge through piston rings and valves.

The quantities $\mathrm{Q}_{\mathrm{ch}}$ and $\mathrm{Q}_{\mathrm{r}}$ cannot be determined in operating conditions due to the lack of required measurable data. Therefore for diagnostic purposes it is advisable to use net heat release characteristics $Q_{n}$.

The formula for heat $\mathrm{dQ}_{\mathrm{n}}$ for perfect gas can be written as:

$$
\mathrm{dQ}_{\mathrm{n}}=(\kappa-1)^{-1}(\mathrm{~V} \mathrm{dp}+\kappa \mathrm{pdV})
$$

where:

$\kappa-$ adiabatic exponent

$\mathrm{V}-$ gas volume

$\mathrm{p}-$ gas pressure.

After dividing equation (3) by the displacement volume, the formula for the net heat release rate as a function of the crankshaft rotation angle takes the form:

$$
q^{\prime}=\frac{d q}{d \alpha}=(\kappa-1)^{-1}\left(\mathrm{v} \frac{\mathrm{dp}}{\mathrm{d} \alpha}+\kappa \mathrm{p} \frac{\mathrm{dv}}{\mathrm{d} \alpha}\right)
$$

where:

$\mathrm{v}-$ dimensionless gas volume [3],

$\alpha-$ crankshaft rotation angle.

Here, the basic unit for the quantity $\mathrm{q}^{\prime}$ is $\left[\mathrm{J} / \mathrm{m}^{3 . \circ} \mathrm{OWK}\right]$.

The net heat release, $\mathrm{q}$, in relation to the stroke volume and as a function of the crankshaft rotation angle, is expressed by the integral:

$$
\mathrm{q}=\int^{\alpha} \mathrm{q}^{\prime} \mathrm{d} \alpha
$$

where the beginning of integration 1s assumed at BDC piston position.

\section{ASSESSING THE EFFECT OF CHANGES OF THE EXPONENT K ON THE ACCURACY OF THE DETERMINED CHARACTERISTICS}

For both the air and the exhaust gas, the $\kappa$ values almost linearly depend on the gas temperature (Fig. 1).

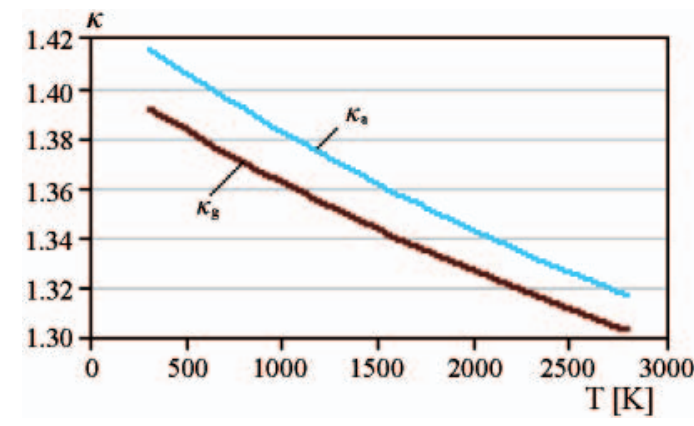

Fig. 1. Adiabatic exponent $\boldsymbol{\kappa}$ vs. temperature $\boldsymbol{T}$. The characteristic was made based on the approximation of tabularised data [7]:

$$
\boldsymbol{\kappa}_{a}-\text { dry air, } \boldsymbol{\kappa}_{g}-\text { exhaust gas }
$$

The effect of changes of the adiabatic exponent $\kappa$ on $\mathrm{q}^{\prime}$ can be taken into account using, for instance, the method of successive approximations. For the first approximation we assume a constant (average) value of $\kappa$. In the next calculation step the fuel consumption and the volume of the produced exhaust gas are assessed based on the heat release determined in the precious calculation step. Although it looks simple, this algorithm would make the calculation process take much longer without delivering any additional diagnostic information. The level or the possible error made when we stop at the first approximation of the characteristics is to be estimated.
The temperatures at characteristic points of the working cycle for ship engines are within the ranges of : 800-1100 K for the end of compression, 1700-2000 K for the end of combustion, and 900-1200 K for the end of expansion [6], which in total covers the temperature range of $800-2000 \mathrm{~K}$. If we assume in the calculations that the value of $\kappa$ is the average calculated for the middle temperature within the above range, then the errors which we make when determining the maximum value of q' do not exceed $\pm 3.5 \%$, and for q they do not exceed $\pm 2 \%$, which was checked on a number of types of ship engines. The scale of this error can be even more reduced (below $1 \%$ ) when we take into account the range of temperatures of the working medium which is characteristic for the examined type of engine and given load.

\section{DEFORMATIONS OF CHARACTERISTICS INTRODUCED BY GAS PASSAGES AND INDICATOR VALVES}

In order to recognise the nature of the effects of the gas passages and indicator valves on the heat release characteristics, a series of tests were performed on the research rig of the medium-speed engine Sulzer 6AL20/24. The arrangement and dimensions of the gas passages and the distribution of pressure sensors are schematically presented in Fig. 2, preserving relevant proportions.

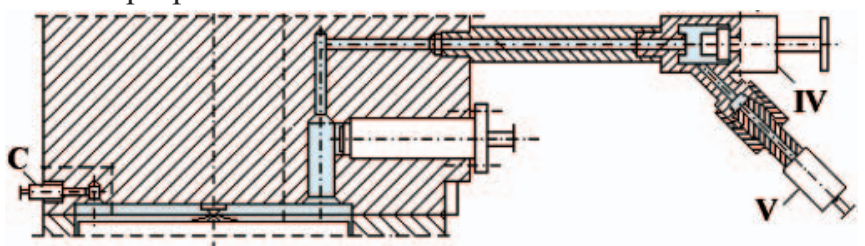

Fig. 2. Scheme of indicator passages in the Sulzer engine 6AL20/24, with the distribution of pressure sensors: $\boldsymbol{I V}$-indicator valve, $\boldsymbol{C}$-Optrand pressure sensor in the cylinder $\boldsymbol{V}$-Kistler pressure sensor on the indicator valve

For technical reasons, the measurements were performed using pressure sensors of two different types and made by two different producers. The measuring paths were calibrated before the measurements and checked after the measurements for $100 \%$ and $50 \%$ of the nominal load of the engine. The sensors were installed on a special connector fixed to the indicator valve in such a way that during the calibration and final check their front plates were opposite to each other, at a distance of $5 \mathrm{~mm}$. Differences in indications in the assumed range of measurement did not exceed $\pm 0.5 \%$ of the maximum value of the measured pressure for the nominal load.

Before calculating the heat release characteristics, the indicator diagrams $\mathrm{p}_{\mathrm{C}}$ and $\mathrm{p}_{\mathrm{v}}$ (Fig. 4) were smoothed using the method of multiple approximation and a third-order power polynomial [2]. Without this procedure the heat release rate curves would not be readable. The smoothing removed (smoothed) the high-frequency measuring noise, $\mathrm{Dp}_{\mathrm{C} 1}$ and $\mathrm{Dp}_{\mathrm{v} 1}$, introduced by the sensors, and the disturbances $\mathrm{Dp}_{\mathrm{C} 2}, \mathrm{Dp}_{\mathrm{v} 2}$ generated by gas oscillation in the passages (Fig. 3).

The above smoothing is necessary to obtain sufficient smoothness of the heat release characteristics. However, it does not remove the deformation of the pressure time-history $\mathrm{p}_{\mathrm{v}}$ on the indicator valve (Fig. 4), caused by gas compression and expansion in the passages, heat transfer, and adding up of the remaining errors introduced by the sensors.

The deformation of the $\mathrm{p}_{\mathrm{v}}$ curve, as compared to the pressure $\mathrm{p}_{\mathrm{C}}$ in the cylinder, was a source of relevant deformations in the heat release characteristics $\mathrm{q}_{\mathrm{V}}^{\prime}$ and $\mathrm{q}_{\mathrm{V}}$, compared to the characteristics of $\mathrm{q}_{\mathrm{C}}^{\prime}$ and $\mathrm{q}_{\mathrm{C}}$ (Fig. 4). 


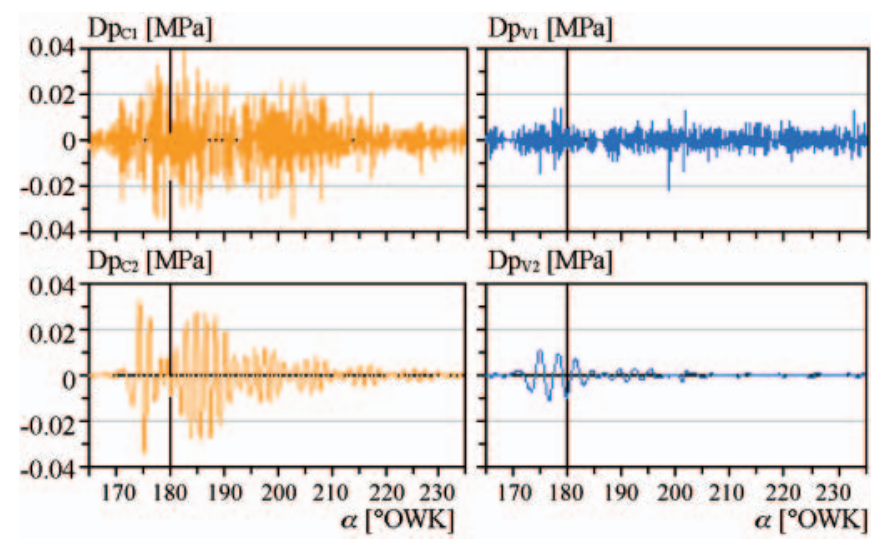

Fig. 3. Disturbances removed (smoothed) from the indicator diagrams after their smoothing using the method of multiple moving approximation and the third-order power polynomial: $\boldsymbol{D} \boldsymbol{p}_{C 1}, \boldsymbol{D} \boldsymbol{p}_{C 2}-$ for $\boldsymbol{p}_{C}$ (Fig. 4) in the cylinder, $\boldsymbol{D} \boldsymbol{p}_{V 1}, \boldsymbol{D} \boldsymbol{p}_{V 2}-$ for $\boldsymbol{p}_{V}$ (Fig. 4) on the indicator valve

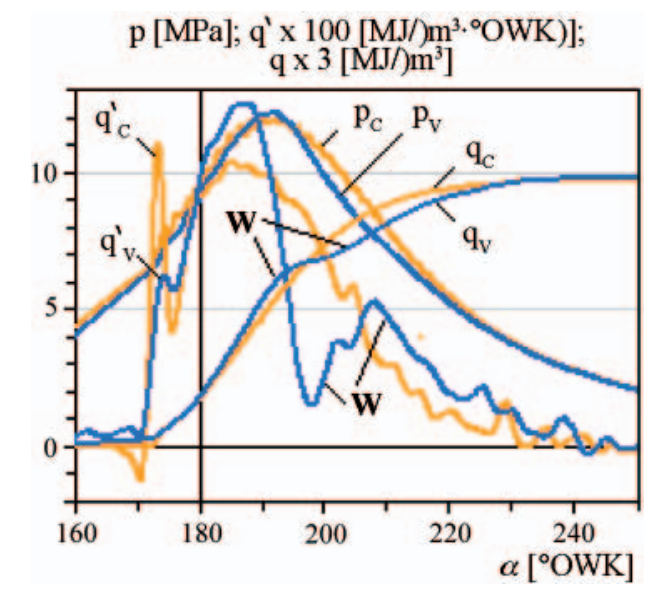

Fig. 4. The effect of gas passages and indicator valves (Fig. 2) on the timehistory of pressure $\boldsymbol{p}_{V}$, and the heat release characteristics $\boldsymbol{q}_{V}^{\prime}$ and $\boldsymbol{q}_{V}$ for nominal load: $\boldsymbol{p}_{C}, \boldsymbol{q}_{C}^{\prime}, \boldsymbol{q}_{C}$-for cylinder, $\boldsymbol{p}_{V}, \boldsymbol{q}_{V}^{\prime}, \boldsymbol{q}_{V}$-for indicator valve, $\boldsymbol{W}$-deformation (waves) observed on $\boldsymbol{q}^{\prime}{ }_{V}$ and $\boldsymbol{q}_{V}$ curves

Along with smaller peak values, characteristic deformations (waves) $\mathbf{W}$ can be observed on the $\mathrm{q}_{\mathrm{v}}^{\prime}$ and $\mathrm{q}_{\mathrm{V}}$ curves. They can also be noticed on the characteristics of medium-speed engines of other types (Fig. 7). On the other hand, this type of deformation does not exist, in practice, on the characteristics recorded on low-speed engines (Fig. 6).

\section{THE EFFECT OF TDC POSITION ESTIMATION ERROR ON THE SHAPES OF CHARACTERISTICS}

It is noteworthy that in the case of the examined engine (Fig. 2) the deformation of the pressure time-history recorded on the indicator valve is accompanied by a pressure signal delay, amounting to $2.7^{\circ} \mathrm{OWK}$, which was removed by shifting the diagrams to their TDC points. In the examined case for the measurement on the indicator valve at nominal load, the TDC position estimation error, which was equal to $\pm 1^{\circ} \mathrm{OWK}$, was a source of errors in determining maximum $\mathrm{q}^{\prime}$ and $\mathrm{q}$ values which approximately amounted to $\pm 7 \%$ (Fig. 5).

For engines of different types, the effect of TDC position estimation error on the maximum values of $\mathrm{q}^{\prime}$ and $\mathrm{q}$ is comparable with its effect on the errors in determining the average indicated pressure. The applied method of TDC position estimation on indicator diagrams secures high accuracy, especially in case of low-speed engines [3].

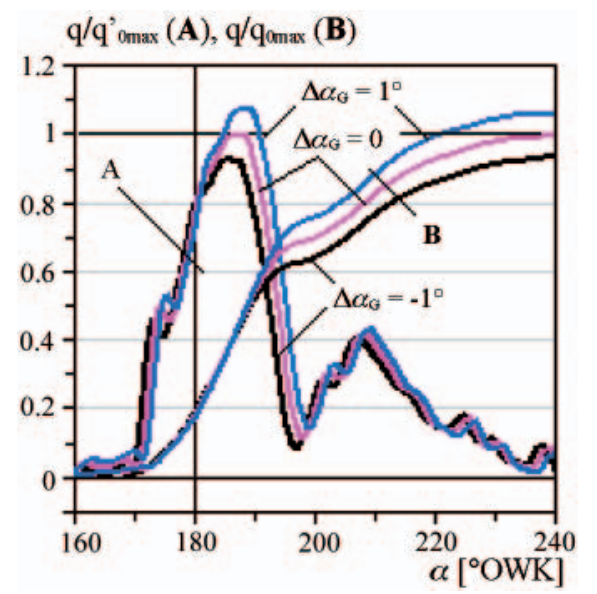

Fig. 5. The effect of the TDC position estimation error $\Delta \alpha_{G}$ on the shapes of heat release characteristics $\boldsymbol{q}_{V}^{\prime}$ and $\boldsymbol{q}_{V}$ (Fig. 4): $q^{\prime}{ }_{\text {Omax }}$ and $q_{0 \max }$ are the maximum values for the reference characteristics $\left(\Delta \alpha_{G}=0\right)$

\section{ASSESSING THE POTENTIAL FOR DIAGNOSTIC APPLICATION OF HEAT RELEASE CHARACTERISTICS}

For a newly built and properly adjusted engine, strong convergence of q' and q characteristics is observed for particular cylinders (Fig. 6).

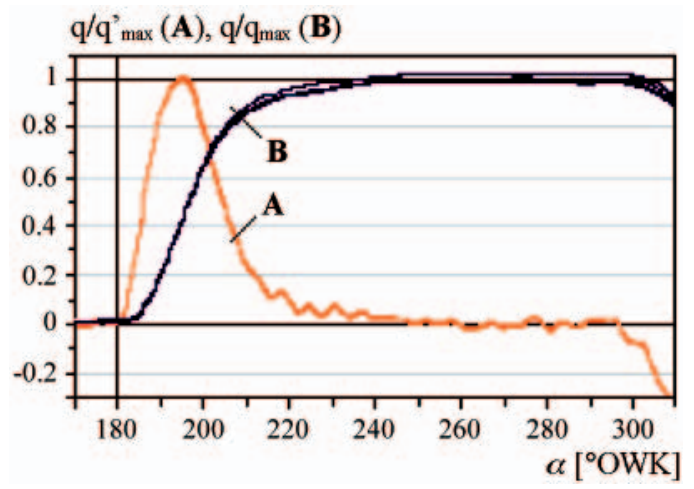

Fig. 6. Comparing heat release characteristics $\boldsymbol{q}^{\prime}$ and $\boldsymbol{q}$ for all 5 cylinders in a newly built low-speed engine 5RTA52 (sea trials): $\overline{\boldsymbol{q}}_{\text {max }}, \overline{\boldsymbol{q}}_{\text {max }}$ (referential values) - maximum $\boldsymbol{q}^{\prime}$ and $\boldsymbol{q}$ values recorded in particular cylinders and averaged for the entire engine

Noteworthy is the coincidence of the waves in particular $\mathbf{A}$ curves (Fig. 6), irrelevant of the location of the measuring point (cylinder). This tendency is also characteristic for other types of low-speed engines and for medium-speed engines.

For an engine after large number of operating hours or an inefficient one, the q' and q characteristics differ between each other depending on the technical condition of fuel system components (Fig. 7).

These characteristics (Fig. 7) were determined after the running repair and the adjustment of the injection system. Remarkable differences in the characteristics are caused by advanced wear of injection system components due to a large number of operating hours without engine renovation.

Based on the heat release characteristics we can make a judgement about the course of the fuel injection and combustion process, and evaluate the accuracy of its adjustment, efficiency and wear of particular injection systems. Additional information can be obtained by analysing trends of selected parameters of the characteristics.

It is advisable to create a catalogue of symptoms (patterns) of typical inefficiencies for engines of certain types. This can be done via observation of engines in operation or simple simulation tests. 


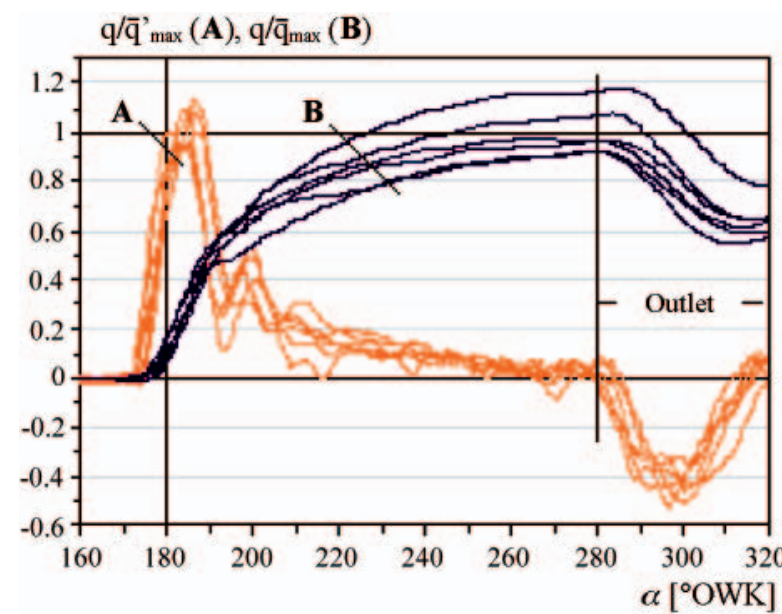

Fig. 7. Comparing heat release characteristics $q^{\prime}$ and $q$ determined for one block of cylinders in the 12-cylinder two-stroke medium-speed engine 40DM ( $n=750 \mathrm{rev} / \mathrm{min}$, nominal load) after large number of operating hours:

$\overline{\boldsymbol{q}}{ }_{\text {max }}, \overline{\boldsymbol{q}}_{\text {max }}$ (referential values) - maximum $q^{\prime}$ and $q$ values averaged for the entire engine, Outlet - exhaust space

The permeability of exhaust ducts, in particular in twostroke engines which burn heavy fuel, can be diagnosed using characteristics parts corresponding to the exhaust space (Fig. 7). Some inadequacy of the adopted heat release model for this space is of no importance here.

\section{CONCLUSIONS}

- The net heat release characteristics can be determined based on ship engine indicator diagrams, with the accuracy sufficient for diagnostic purposes.

- For low-speed engines, deformations of the characteristics introduced by passages and indicator valves are negligible. For medium-speed engines the deformations of pressure time-histories are large but repeatable, which provides opportunities for their use for diagnostic purposes.

- Based on the heat release characteristics one can conclude about the range and uniformity of load distribution over particular cylinders, condition of the control, correctness of work and technical condition of the injection system, as well as on the level of exhaust gas resistance.

- It is advisable to create a catalogue of inefficiency symptoms, which will help ship engineers to detect and recognise inefficiencies based on the heat release characteristics.
NOMENCLATURE

(repeated symbols which need defining)

TDC - top dead centre (of a piston)

BDC - bottom dead centre (of a piston)

$\mathrm{p}_{\mathrm{C}} \quad-$ pressure (indicator diagram) measured in the cylinder

$\mathrm{p}_{\mathrm{v}} \quad-$ pressure (indicator diagram) measured on the indicator valve

$\mathrm{q}^{\prime} \quad-\quad$ net heat release rate related to the stroke volume, $\left[\mathrm{J} / \mathrm{m}^{3 . \circ} \mathrm{OWK}\right]$

$\mathrm{q} \quad-\quad$ net heat release related to the stroke volume, $\left[\mathrm{J} / \mathrm{m}^{3 . \circ} \mathrm{OWK}\right]$

$\mathrm{q}_{\mathrm{C}}^{\prime}, \mathrm{q}_{\mathrm{C}}-\mathrm{q}^{\prime}$, q determined for the indication in the cylinder

$\mathrm{q}_{\mathrm{v}}^{\prime}, \mathrm{q}_{\mathrm{V}}-\mathrm{q}^{\prime}$, $\mathrm{q}$ determined for the indication on the indicator valve

${ }^{\circ} \mathrm{OWK}$ - degree of crankshaft rotation angle (crank angle).

\section{BIBLIOGRAPHY}

1. Heywood J. B.: Internal Combustion Engine Fundamentals. McGraw-Hill Book Company, 1988.

2. Polanowski S.: Application of movable approximation and wavelet decomposition to smoothing-out procedure of ship engine indicator diagrams. Polish Maritime Research, No. $2 / 2007$

3. Polanowski S.: Determination of location of Top Dead Centre and compression ratio Valle on the basis of ship engine indicator diagram. Polish Maritime Research, No. 2/2008

4. Rychter T., Teodorczyk A.: Mathematical modelling of piston engine working cycle (in Polish). PWN, Warsaw 1990.

5. Schweitzer P.: The Tangent Method of Analysis of Indicator Cards of Internal Combustion Engines. Biulletin No. 35, Pensylvania State University, September 1926.

6. Wanszejd W. A.: Sudowyje dwigatieli wnutrienniewo sgoranija. Sudostrojenie, Leningrad 1977.

7. Wiśniewski S.: Fundamentals of internal combustion engine thermodynamics (in Polish). WNT, Warsaw 1962.

\section{CONTACT WITH THE AUTHOR}

Prof. Stanisław Polanowski

Mechanic-Electric Faculty,

Polish Naval University Śmidowicza 69

81-103 Gdynia, POLAND

e-mail: SPolanowski@o2.pl 